\title{
FORMULATION AND OPTIMIZATION OF QUERCETIN LOADED NANOSPONGES TOPICAL GEL: EX VIVO, PHARMACODYNAMIC AND PHARMACOKINETIC STUDIES
}

\section{Y. SARAH SUJITHA, Y. INDIRA MUZIB*}

\author{
Institute of Pharmaceutical Technology, Sri Padmavathi Mahila Visvavidyalayam, (Women's University), Tirupati, India
}

Email: suji.sarah@gmail.com

Received: 28 Feb 2019, Revised and Accepted: 04 Jul 2019

\begin{abstract}
Objective: Quercetin is therapeutically hampered because of its poor solubility. The present investigation was aimed to prepare quercetin loaded nanosponges topical gel to enhance the solubility and efficacy of the drug.

Methods: Quercetin nanosponges were prepared by emulsion solvent diffusion method. Developed nanosponges optimized by particle size, SEM, entrapment efficiency, FT-IR, DSC, P-XRD, In vitro studies. The optimized formulation of nanosponges was loaded into a topical gel and it was characterized by ex-vivo, in vivo Pharmacodynamic and kinetic studies.

Results: The particle size and zeta potential of optimized nanosponges were found to be $188.3 \mathrm{~nm}$ and- $0.1 \mathrm{mV}$. Surface morphology was studied using SEM Analysis which showed tiny sponge-like structure and entrapment efficiency was found to be $96.5 \%$. In vitro drug release of optimized nanosponges was found to be $98.6 \%$ for 7 hours. Optimized nanosponges entrapped gel was prepared by using carbopol 934 and hydroxypropyl methylcellulose as gelling agents. The prepared nanogels were homogenous and ex-vivo skin permeation studies of the optimized nanosponges gel was found to be $98.1 \%$ for $5 \mathrm{~h}$, quercetin loaded nanosponges has shown higher skin permeation efficiency $\left(18.4 \mu \mathrm{g} / \mathrm{cm}^{2} \pm 2.1\right) \mathrm{compared} \mathrm{to} \mathrm{pure}$ quercetin gel. The pharmacokinetic and pharmacodynamic studies showed that the quercetin loaded nanosponges has shown more effective when compared to marketed formulation.
\end{abstract}

Conclusion: Quercetin loaded nanosponges gel has shown a significant increase in activity $(\mathrm{p}<0.05)$ compared to the marketed formulation (Voveran Emulgel).

Keywords: Nanosponges, Nanosponges gel, Quercetin, In vitro, Ex-vivo studies, In vivo studies

(C) 2019 The Authors. Published by Innovare Academic Sciences Pvt Ltd. This is an open-access article under the CC BY license (http://creativecommons.org/licenses/by/4.0/) DOI: http://dx.doi.org/10.22159/ijap.2019v11i5.32850

\section{INTRODUCTION}

The plant and plant parts are used for therapeutic properties like Dacus carota, Ocimum sanctum, Curcuma domestica valet on, Glycyrrhiza glabra, Foeniculum vulgar, Cuscuta reflexa and Withinia sommifera were known as the best examples for anti-inflammatory, antidiabetic, and anti-hypercholesterolemia [Soon am park et al.; 2013, Wang Q et al.;2018]. Flavonoids are a group of plant metabolites found in a variety of fruits and vegetables, which are 15 carbon atom phenolic molecules. Quercetin is one of the vital bioflavonoid present in more than twenty plant materials which is known for its therapeutic activities like anti-inflammatory, antihypertensive, antidiabetic, antioxidant and anti-obesity [Qi Tan, Weidong Liu, et al;;2011]. Quercetin name was derived from the Latin word quercetum means oak forest belonging to the class flavanols. Quercetin is chemically 2(3,4-dihydroxyphenyl)-3,5,7-trihydroxychromen-4-one, having one OH group attached at positions $3,5,7,3^{1}$, and $4^{1}$ positions [Soheirn. abdel rehaman et al. 2014]. Quercetin is yellow in color which is sparingly soluble in water and completely soluble in alcohol, which belongs to BCS class II drugs [Chen-Yu G et al.; 2012]. It is also available as an abundant dietary supplement present in the red grapes, onions, buckwheat, bark, olive oil, green tea, red wine, cranberries [Gulian quan et al:2015]. Quercetin has a most remarkable property of anti-inflammatory conditions as it stops the formation of prostaglandins, cyclooxygenase (COX) as well as histamine release. Quercetin is mostly consumed orally, but the drawback is that it has low oral bioavailability [7]. The absorption of quercetin was low in the gastrointestinal tract due to its poor solubility. Quercetin was enzymatically hydrolyzed and it was glucuronidated or converted into the sulfated form of quercetin when taken orally [8]. The bioavailability of this quercetin was nearly $44 \%$ when taken orally because most of the drug metabolized by the liver and is eliminated from the intestinal cells through an efflux mechanism [8]. So the topical drug delivery system has the advantage to overcome these problems [9]. The structure and composition of the drug were the major barriers for this type, but the formulation of the polymer type drug delivery can overcome this problem $[10,11]$. Nanosponge has a 3-dimensional structure with tiny channels and mesh-like structure on the surface, when the solution is mixed with the polymers it forms a mesh-like structure, with naturally biodegradable polymer as the backbone which results in the formation of pockets like structure in which drug can be loaded [12]. Physically these nanosponges are in the form of dry powder which can be formulated as orally, topically and parentally. A wide variety of drugs can be loaded into the nanosponges but the drug molecular weight should be less than 400 daltons [13]. Nanosponges due to its nano size can be successfully incorporated into the topical gel, effectively releases the drug with increased stability, bypasses the first pass metabolism and reduces the gastric irritation. Due to its small size, they can freely circulate into the blood until they get attached to the specific target site. Hence the objective of the present study is to increase the solubility of poorly aqueous soluble quercetin by formulating quercetin into nanosponges. Quercetin containing nanosponges loaded into the gel was characterized by in vitro drug release, in vivo pharmacokinetic and pharmacodynamic studies.

\section{MATERIALS AND METHODS}

\section{Materials}

Quercetin, Ethylcellulose, Polyvinyl alcohol was purchased from SD. Fine-chemicals Ltd, Dichloromethane was purchased from Merck limited, Mumbai, Hydroxypropyl methylcellulose (HPMC), Carbopol 934, HPLC methanol was purchased from HiMedia. Triethanolamine, Glycerine and Methylparaben and Propylparaben have purchased from Merck limited, Mumbai. Microorganisms (Staphylococcus aureus, E. coli, Proteus vulgaris, Bacillus subtilis) obtained from HiMedia.

\section{Methods}

Preparation of quercetin-loaded nanosponges

Quercetin loaded nanosponges were formulated by using the emulsion solvent diffusion method using ethyl cellulose as polymer 
and quercetin as a drug. Required quantities of the drug and polymer were added into the solvent dichloromethane and mixed well. The above solution was added into the external aqueous phase containing required quantities of polyvinyl alcohol and $150 \mathrm{ml}$ of water $[14,15]$ in a continuous stirring process at $1200 \mathrm{rpm}$ for 30 min on a magnetic stirrer. The formed nanosponges were filtered and dried in the oven. The formulation composition of quercetinloaded nanosponges was given in table 1 .

Table 1: Formulation composition of quercetin-loaded nanosponges

\begin{tabular}{|c|c|c|c|c|}
\hline S. No. & Formulation code & Drug: Ethyl cellulose (mg) & PVA(mg) & Water(ml) \\
\hline 1 & F1 & $1: 1$ & 100 & 150 \\
\hline 2 & $\mathrm{~F} 2$ & $1: 2$ & 100 & 150 \\
\hline 3 & F3 & $1: 3$ & 100 & 150 \\
\hline 4 & F4 & $1: 4$ & 100 & 150 \\
\hline 5 & F5 & $1: 5$ & 100 & 150 \\
\hline 6 & F6 & $1: 4$ & 100 & 200 \\
\hline 7 & F7 & $1: 4$ & 100 & 300 \\
\hline 8 & F8 & $1: 4$ & 150 & 150 \\
\hline 9 & F9 & $1: 4$ & 200 & 150 \\
\hline
\end{tabular}

\section{Quercetin loaded nanosponges gel}

A nanosponge showing a high percentage of drug release F4 was converted into a topical gel using polymers carbopol 934 and hydroxypropyl methylcellulose (HPMC). Carbopol934 and hydroxypropyl methylcellulose [16] were weighed and soaked for
$24 \mathrm{~h}$ in the required quantity of water and stirred using a mechanical stirrer at $1000 \mathrm{rpm}[16,17]$. In the above-formed gel required quantities of glycerin, methylparaben, propylparaben, and triethanolamine were added with continuous and slow stirring for $30 \mathrm{~min}$. Composition of quercetin-loaded nanosponges gel was given in table 2.

Table 2: The composition of topical gels using different concentrations of polymers (Carbopol 934 and HPMC)

\begin{tabular}{llll}
\hline S. No. & Formulation code & Composition & Ratios of polymers \\
\hline 1 & X1 & Carbopol: HPMC & $1: 1$ \\
2 & X2 & Carbopol: HPMC & $1: 2$ \\
3 & X3 & Carbopol: HPMC & $1: 3$ \\
4 & X5 & Carbopol: HPMC & $1: 4$ \\
5 & X6 & Carbopol: HPMC & $2: 1$ \\
6 & & Carbopol: HPMC(pure) & $1: 1$ \\
\hline
\end{tabular}

\section{Evaluation of quercetin-loaded nanosponges}

\section{Scanning electron microscopy}

Scanning electron microscopy (JOEL-JSM-IT500) was used for surface morphology and structural elucidation of the formed nanosponges. The formed images were captured, particles size and surface morphology were studied $[18,19]$.

\section{Particle size and zeta potential}

The particle size and zeta potential of the formed quercetin loaded nanosponges were determined by using the Horiba zeta sizer [20]. The dispersion was mixed, thoroughly diluted with water and then analyzed. Each sample was analyzed in triplicates and the average of the three values was noted.

\section{Differential scanning calorimeter}

Differential scanning calorimeter (Metler Toledo, Switzerland) studies were done to find out the chemical compatibility between the polymers and drug. For this, the melting point of the pure drug was compared to the melting point of the drug in the formulation $[21,22]$. The instrument was calibrated with tin as standard material. $5 \mathrm{mg}$ of the sample was taken in an aluminum crucible and heated at a rate of $10^{\circ} \mathrm{C}$ in the temperature range of $100-300^{\circ} \mathrm{C}$.

\section{$\mathrm{X}$-ray diffraction studies}

The crystalline nature of the drug in the pure quercetin and quercetin loaded nanosponges was evaluated by using these P-XRD studies [23, 24]. Powder X-ray diffractometer (Regaku-mini plus 500 ) was done by using k-beta (x2) filtered, $\mathrm{Cu}-\mathrm{k}$, alpha radiation, and at a voltage of $30 \mathrm{kv}$, and $15 \mathrm{~mA}$.

\section{Drug compatibility with excipients}

Drug compatibility study of pure quercetin, ethylcellulose, and nanosponges formulated using quercetin was carried out by potassium bromide pellets by applying pressure of $100 \mathrm{~kg}$ to form a pellet $[25,26]$. They formed pellet was scanned in the region of 4000$600 \mathrm{~cm}^{-1}$ using (FTS300 Spectrophotometer (Glab), Canton, MA).

\section{Entrapment efficiency}

The amount of quercetin present in the quercetin loaded nanosponges was determined by taking $10 \mathrm{mg}$ of quercetin-loaded nanosponges in $10 \mathrm{ml}$ of phosphate buffer $\mathrm{pH} 7.4$ and kept aside for $24 \mathrm{~h}$ and then after $24 \mathrm{~h}$ the solution was stirred using a magnetic stirrer (Remi equipment, Mumbai) at $1000 \mathrm{rpm}$ for $30 \mathrm{~min}$ and filtered. The filtered solution absorbance was measured by using a UV visible spectrophotometer (Shimadzu, Japan) at $372 \mathrm{~nm}$.

\section{In vitro drug release studies}

The release rates of quercetin-loaded nanosponges were investigated using the dialysis membrane, which is having a pore size of $2.4 \mathrm{~nm}$ and a molecular weight of 12,000-14,000 daltons was used. This dialysis membrane was soaked $12 \mathrm{~h}$ before using in distilled water. $100 \mathrm{ml}$ of phosphate buffer of $\mathrm{pH} 7.4$ was taken in a beaker $[26,27]$. The beaker was placed on a magnetic stirrer (Remi equipment, Mumbai) at $100 \mathrm{rpm}$ and at a temperature of $37^{\circ} \mathrm{C}$. An aliquot of $5 \mathrm{ml}$ of the sample was taken at regular intervals; an equal amount of fresh buffer was replaced in the beaker to maintain sink conditions [28]. The samples were measured using UV-visible spectrophotometer (Shimadzu, Japan) at a wavelength of $372 \mathrm{~nm}$.

\section{Characterization of quercetin-loaded nanosponges gel}

\section{Physical examination of gel}

The formed quercetin loaded nanosponges gel was physically tested for the presence of any aggregates and uniformity by visually after it was stored in a container.

\section{The $\mathrm{pH}$ of the gel}

The $\mathrm{pH}$ of the quercetin loaded nanosponges gel was determined by using a digital $\mathrm{pH}$ meter (Remi equipment, Mumbai). 


\section{Viscosity}

The viscosity of the gel was determined by Brookfield LV Viscometer [29]. The prepared quercetin loaded nanosponges gel of $5 \mathrm{~g}$ was taken in a beaker and by selecting spindle number 64 the viscosity of the gel was noted.

\section{Percentage of drug content}

$10 \mathrm{mg}$ of the gel was placed in $10 \mathrm{ml}$ of phosphate buffer of $\mathrm{pH} 7.4$ and the solution was kept aside for $24 \mathrm{~h}$ and the solution was stirred on a magnetic stirrer (Remi equipment, Mumbai) for $30 \mathrm{~min}$ and filtered [29]. The absorbance of a filtered solution was measured by using a UV spectrophotometer (Shimadzu, Japan) at $372 \mathrm{~nm}$.

\section{Antimicrobial activity by a zone of inhibition method}

The $20 \mathrm{ml}$ of sterilized broth was taken individually into four Petri dishes and inoculated with four different types of bacteria (Staphylococcus aureus, E. coli, Proteus vulgaris, Bacillus subtilis). The subcultured bacteria were spread on the solidified nutrient broth and four wells were kept on the solidified agar medium of $6 \mathrm{~mm}$ to place quercetin loaded nanosponges gel [30]. The Petri dishes were incubated at $35-37{ }^{\circ} \mathrm{C}$ for $24 \mathrm{~h}$. Then the zone of inhibition of microbial growth was measured using the scale in centimeters.

\section{Ex-vivo skin permeation studies and flux value determination}

The ex-vivo skin permeation studies were performed using goatskin in the modified Franz diffusion cell. Goatskin, which was brought from a local slaughterhouse, the hair on the goatskin was removed by using the razor and the skin was wiped with isopropyl alcohol. Goatskin was mounted in between donor and receptor compartment. The donor compartment of the diffusion cell was placed with $500 \mathrm{mg}$ of quercetinloaded nanosponges gel and the receptor compartment was filled with phosphate buffer of $\mathrm{pH} 7.4[31,32]$. The entire setting was placed on the magnetic stirrer (Remi equipment, Mumbai) with an rpm of 100 and temperature $37^{\circ} \mathrm{C}$ was maintained. Then aliquot of $5 \mathrm{ml}$ of the sample was taken at regular intervals of time $0,0.25,0.5,1,2,3,4,6,8,12,24 \mathrm{~h}$ and a freshly prepared buffer of $5 \mathrm{ml}$ was placed back into the receptor compartment to maintain the sink conditions. The samples were measured using the UV spectrophotometer (Shimadzu, Japan) at 372 $\mathrm{nm}$. The obtained results were subjected for the skin permeation parameters like flux, permeation coefficient, lag time and diffusion coefficient by plotting a graph between percentage cumulative drug release on the $\mathrm{y}$-axis and time on the $\mathrm{x}$-axis. The data obtained were also subjected to mathematical models like zero order, first order, Higuchi, Korsmeyer-peppas model [33].

\section{Stability studies}

According to the International Council for Harmonization of technical requirements for pharmaceutical for human use (ICH) guidelines, the stability studies of the prepared quercetin loaded nanosponges gel and quercetin pure gel were conducted. The prepared gels were studied under two variations $25 \pm 2{ }^{\circ} \mathrm{C}$ (refrigerator temperature) and $40 \pm 2$ ${ }^{\circ} \mathrm{C}$ (accelerated stability condition) for a period of three months [34, 35]. Parameters like $\mathrm{pH}$, drug content, homogeneity, in vitro drug release studies were conducted.

\section{In vivo anti-inflammatory activity}

Anti-inflammatory activity studies were carried in male Wistar rats. Animals were procured from Sri Venkateswara Enterprises, Bangalore. The animals were housed under the standard environmental conditions like humidity $(55 \pm 5 \%)$ ambient temperature $\left(25 \pm 1{ }^{\circ} \mathrm{C}\right)$ and $12 / 12 \mathrm{~h}$ dark and light cycles. All the protocols and experimental procedures which were used for the anti-inflammatory study were as per the guidelines of a committee for the purpose of control and supervision of experiments on animals (CPCSEA) and which was approved by institutional animal ethics committee bearing Reg. No.1677/PO/Re/ S/2012/CPCSEA/IAEC/25 dt.3.05.2018. The in vivo pharmacodynamic activity was performed to find out the efficacy of the quercetin loaded nanosponges gel in animals. For this study male Wistar albino rats were used. They were grouped into five and all the animals were kept at an optimum weight of 150-200g and age of $10-12 \mathrm{w}[35,36]$. Group I was treated as the control without any treatment, Group II was normal with only carrageenan $(1 \% \mathrm{w} / \mathrm{v})$ inducer, Group III was treated with marketed Voveran gel, Group IV with pure quercetin gel and Group $\mathrm{V}$ with quercetin loaded nanosponges gel. Carrageenan solution (1\%w/v in normal solution) was used as an inducer to produce inflammation. The animals were kept in observation for 10 min for any behavioral changes. The thickness of the paw was measured $(\mathrm{mm})$ at regular time intervals as $0,30 \mathrm{~min}, 1 \mathrm{hr}, 2 \mathrm{hr}, 3 \mathrm{hr}, 4 \mathrm{hr}$ after inducing carrageenan to plantar region of the left hind paw, quercetin loaded nanosponges gel and pure quercetin gel was applied to the sole of the paw where the inflammation was induced. The paw volume was noted using plethysmometer [37]. The percentage of inhibition of paw volume of rats was calculated by using the formula.

$$
\% \text { inhibition of edema }=\mathrm{V}_{\text {control- }} \mathrm{V}_{\text {test }} / \mathrm{V}_{\text {control }}
$$

\section{In vivo pharmacokinetic study}

Pharmacokinetic parameters were done to estimate the concentration of the drug in the blood. Three groups of rats were taken each group containing randomly six rats, which were housed under normal conditions; the rats were fastened overnight before the experiment with free access to water. The study was conducted with three groups Group I control, Group II for the pure quercetin gel and Group III for quercetin loaded nanosponges gel. Drug concentration was estimated by RP-HPLC method using methanol: orthophosphoric acid (65:35) as a mobile phase, with glibenclamide an internal standard and $\mathrm{C}_{18}$ as a column having dimensions $(4.6 \times 250 \mathrm{~mm}, 5 \mu \mathrm{m})$ (Shimadzu, Japan) with a flow rate of 0.8 $\mathrm{ml} / \mathrm{min}[38,39]$. Blood samples were collected at different time intervals from male Wistar albino rats from retro-orbital into Eppendorf tubes containing sodium citrate as an anticoagulant.

\section{Treatment of plasma samples}

The working solution containing the plasma samples spiked with various concentrations of the quercetin with a fixed amount of internal standard was transferred into a clean centrifugation tube. To this $400 \mu \mathrm{l}$ of methanol was added which acts as a protein precipitant and the solution was vortex mixed and centrifuged at $5000 \mathrm{rpm}$ (Remi equipment, Mumbai) for five minutes. The supernatant solution was separated and injected into an HPLC column using a Hamilton syringe and analyzed at the wavelength of $372 \mathrm{~nm}$ [40].

\section{RESULTS AND DISCUSSION}

\section{Scanning electron microscopy}

The photographs of quercetin-loaded nanosponges using scanning electron microscope are shown in fig. 1(a) to fig. 1(c). The formed nanosponges were spherical in shape with tiny mesh-like structure. The size and shape were unaffected even after encapsulating the quercetin drug in the nanosponges.

\section{Particle size and zeta potential}

All measurements were performed in triplicates for measuring the size and zeta potential of the particles at a temperature of $25 \pm 2{ }^{\circ} \mathrm{C}$ [20]. The dispersions were diluted by using double distilled water to ensure that scattering of light was within the range of sensitivity. The average particle size of the optimized nanosponges was found to be $188.3 \mathrm{~nm}$ with a polydispersity index of 0.13 , which indicates that it has a homogenous particle distribution. Homogeneity of the particles was measured by the polydispersity index, which varies from 0 to 1 the value closer to zero indicates more homology between the particles. Zeta potential of the optimized nanosponges was found to be- $0.1 \mathrm{mV}$. This indicates that the formed quercetin loaded nanosponges were stable through electrostatic repulsion and thereby avoiding the repulsion between the particles. The concentration of the polymer plays an important role in the size of the nanosponges from F1 to F5 (1.1 $\mathrm{nm}, 17.9 \mathrm{~nm}, 18.2 \mathrm{~nm}, 188.3 \mathrm{~nm}, 289.9 \mathrm{~nm}$ ) as the concentration of the polymer increases with respect to the drug the size of the nanosponges increases. Thus polymer and drug ratio plays an important role in particle size analysis. The zeta size fig. 2(a) and potential for F1 to F5 $(-22.0 \mathrm{mV},-11.9 \mathrm{mV},-18.3 \mathrm{mV},-0.1 \mathrm{mV},-3.6 \mathrm{mV})$ was shown in fig. 2(b). 

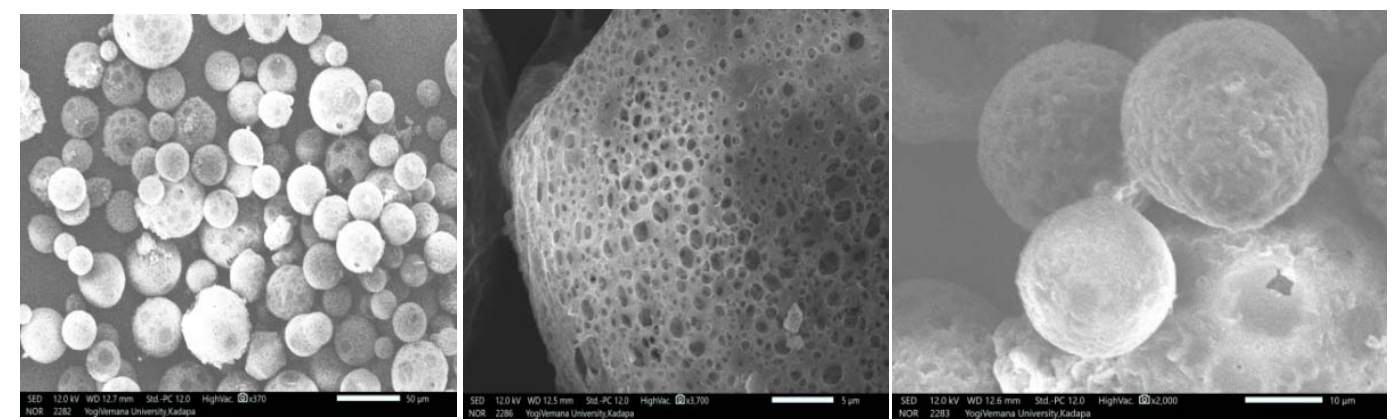

Fig. (1a, 1b, 1c): Scanning electron microscopy images

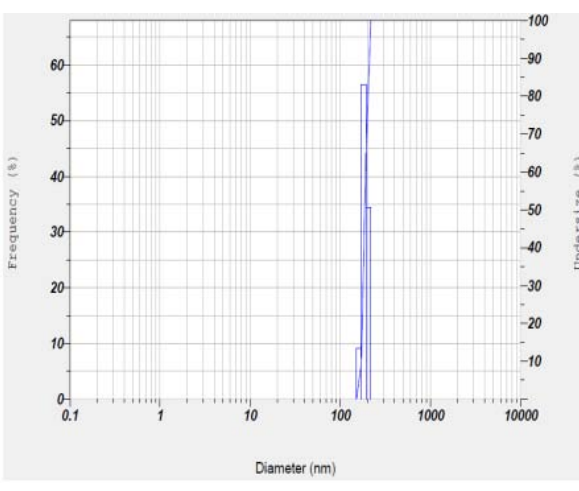

(a)

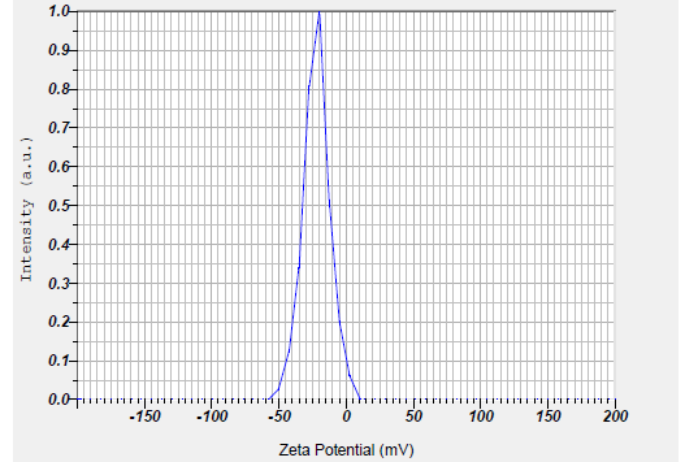

(B)

Fig. (2a, 2b) Zeta size and zeta potential

\section{Differential scanning calorimetry (DSC)}

The Differential scanning calorimetry (DSC) thermograms showed that compatibility between polymer and drug. The thermograms showed the melting point range of $303.6^{\circ} \mathrm{C}$ for pure quercetin drug and $297.85^{\circ} \mathrm{C}$ for the quercetin loaded nanosponges. Both the peaks in thermogram are same, which showed that quercetin loaded nanosponges have the same crystalline state of quercetin in pure form [22]. The DSC thermograms of pure quercetin fig. 3 (a) and quercetin loaded nanosponges were shown in the fig. 3(b).

\section{$X$-Ray diffraction studies}

P-X-ray diffractograms of the pure quercetin and the quercetin loaded nanosponges were taken and analyzed [24]. The diffractograms of the pure quercetin showed $2 \theta$ values as follows $(12.46,14.14,15.73,16.43,18.14,19.9,20.8,25.05,27.8,28.4,30.8)$. This implies that the drug has high crystalline nature and the quercetin loaded nanosponges also show similar $2 \theta$ values in quercetin loaded nanosponges, they both exhibit the same intense sharp peaks. Which indicates the crystalline nature of the quercetin was not lost in the quercetin loaded nanosponges when compared to the pure form. The slight difference is only due to the reduction in size, which implicates the enhancement in the dissolution and solubility of the nanosponges. X-ray diffractogram of pure quercetin fig. 4(a) and quercetin nanosponges were shown in fig. 4(b).

\section{Drug-excipient compatibility study}

Fourier infrared spectroscopy (FT-IR) was one of the most quickened and efficient identifiers of the encapsulated chemical molecules [15]. The characteristic peak intensities of pure quercetin molecules are as follows it shows $\mathrm{O}-\mathrm{H}$ stretching at $3400 \mathrm{~cm}^{-1},=\mathrm{C}-\mathrm{H}$ stretch at $2930 \mathrm{~cm}^{-1}$, $\mathrm{C}=0$ stretching at $1680 \mathrm{~cm}^{-1}$, aromatic $\mathrm{C}=\mathrm{C}$ stretch at $1510 \mathrm{~cm}^{-1}$ and $1610 \mathrm{~cm}^{-1}$ and aromatic C-O stretch at $1220 \mathrm{~cm}^{-1}$. The pure quercetin drug and the quercetin loaded nanosponges have shown the same characteristic peaks which implicate there is no chemical interaction between the polymer and drug. An (FT-IR) Fourier infrared spectroscopy spectrum of pure quercetin fig. 5(a), ethylcellulose fig. 5(b) and optimized quercetin nanosponges were shown in fig. 5(c).

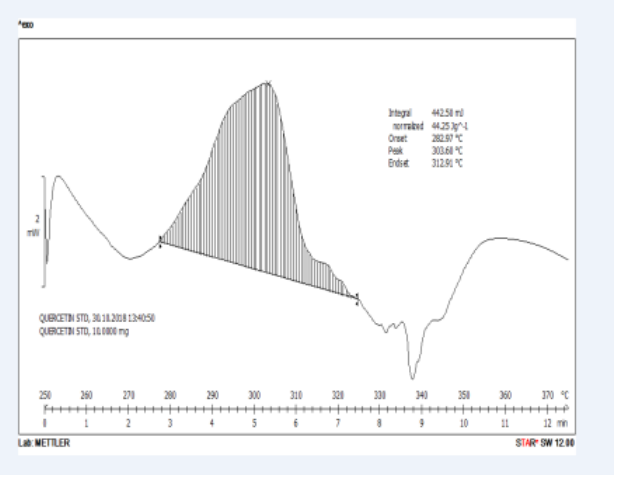

Quercetin pure drug (3a)

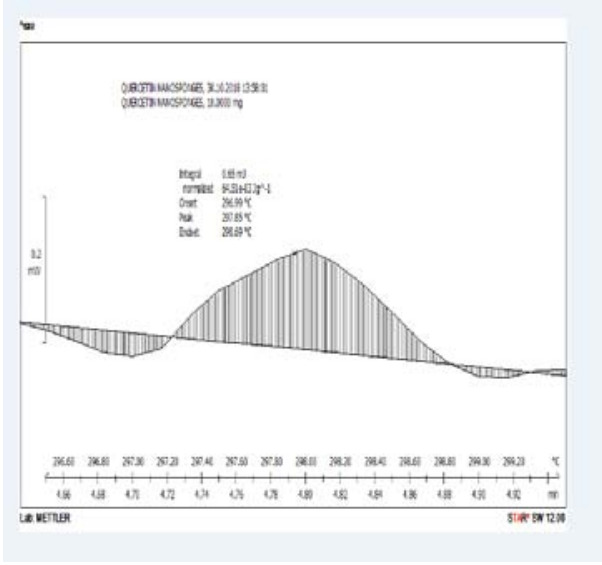

Quercetin loaded nanosponges (3b)

Fig. (3a, 3b): DSC differential scanning calorimetry thermograms 


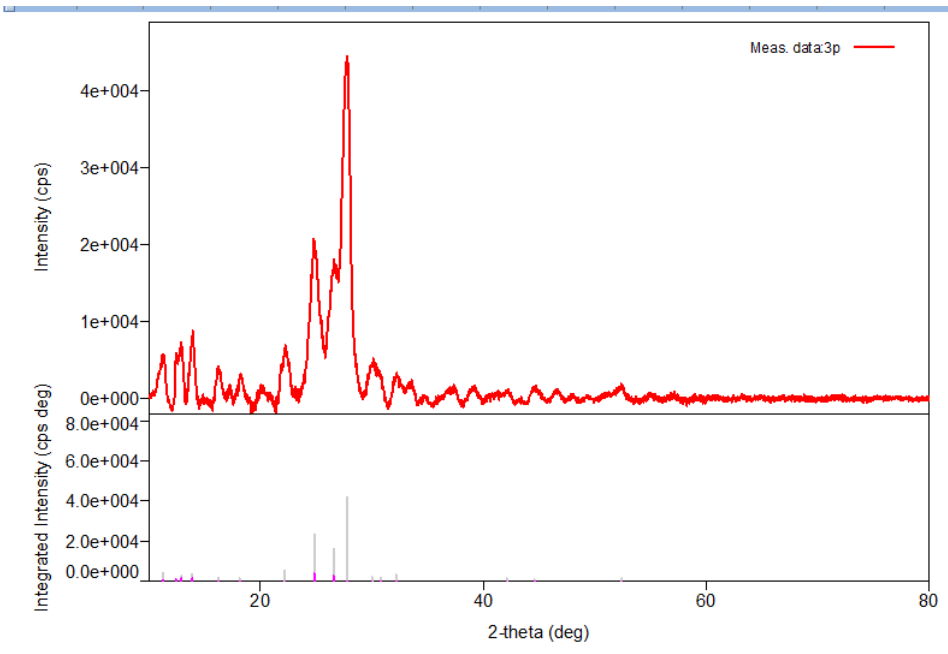

Diffractogram of pure quercetin (4a)

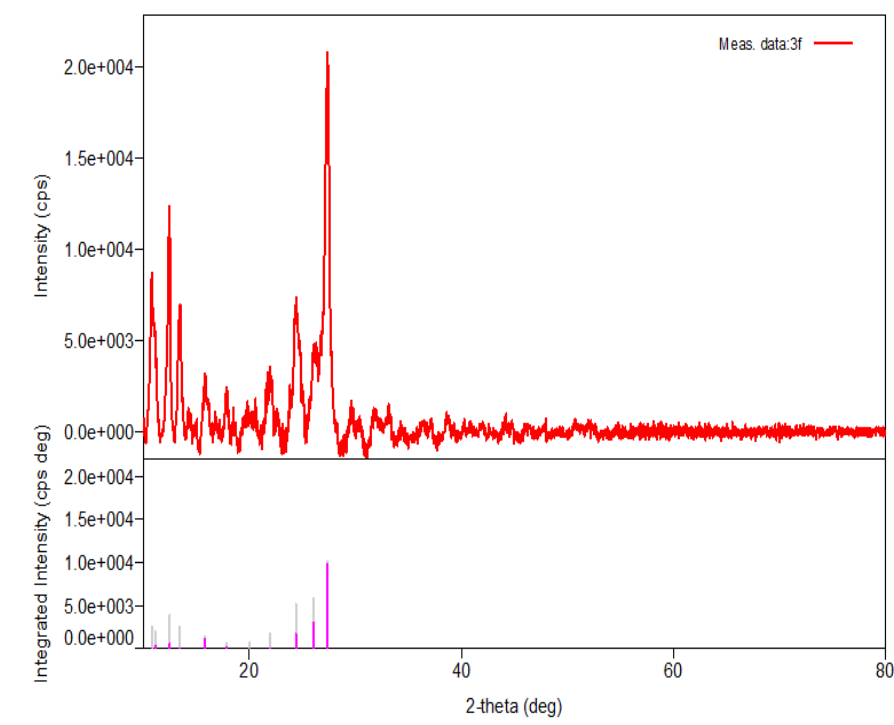

Diffractogram of quercetin loaded nanosponges (4b)

Fig. 4: X-ray diffractogram of pure quercetin (4a), quercetin loaded nanosponges (4b)

\section{Entrapment efficiency}

Entrapment efficiency was measured for all the formulations from F1-F9 the amount of drug entrapped in the nanosponges was found to be in the range of $90-97 \%$ from F1-F5 as the drug entrapped was reduced from F6-F9 this is because as the volume of water and the quantity of polyvinyl alcohol was increased the nanosponges formed were irregular in size and shape and lead to the formation of aggregates and entrapment efficiency is also decreased. Formulations from F6-F9 were discarded for further studies as their entrapment efficiency is very low. The entrapment efficiency of various formulations from F1-F9 was tabulated in table 3 .

Table 3: Entrapment efficiency of quercetin loaded nanosponges

\begin{tabular}{lllll}
\hline S. No. & Formulation & Entrapment efficiency (\%) & Zeta size(nm) \\
\hline 1 & F1 & $96 \pm 1.3$ & 1.1 & Zeta potential (mV) \\
2 & F2 & $98 \pm 1.67$ & 17.9 & -0.1 \\
3 & F3 & $100 \pm 2.5$ & 18.2 & -22.0 \\
4 & F4 & $98 \pm 1.3$ & 188.3 & -18.3 \\
5 & F5 & $97 \pm 1.4$ & 289.9 & -11.9 \\
6 & F6 & $23.5 \pm 1.1$ & - & -3.6 \\
7 & F7 & $9.5 \pm 0.99$ & - & - \\
8 & F8 & $15 \pm 2.13$ & - & - \\
9 & F9 & $10 \pm 0.51$ & - & \\
\hline
\end{tabular}

The values are given as mean $\pm S D, n=3$ 


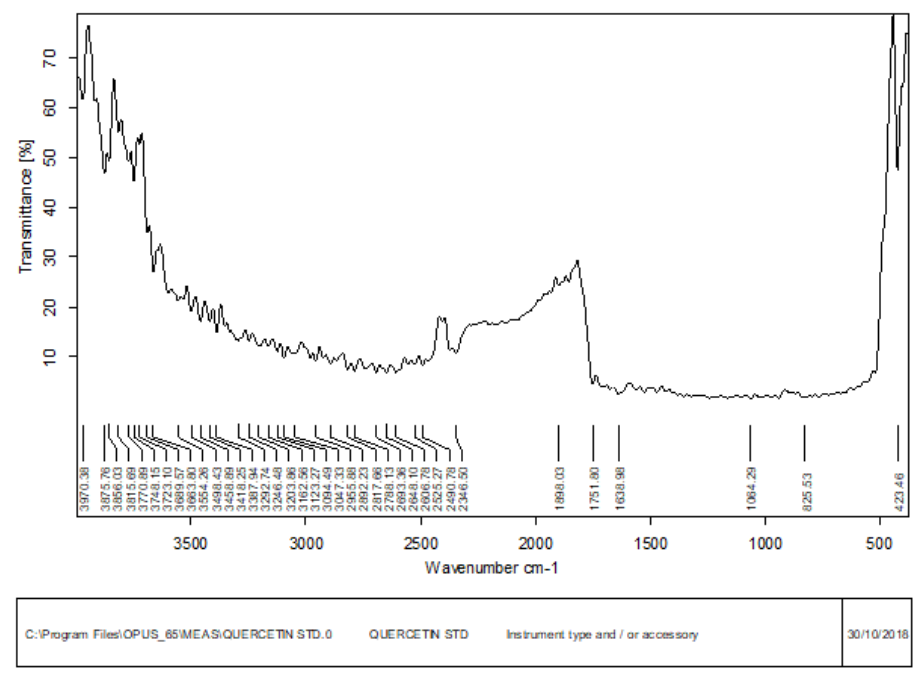

Pure quercetin (5a)

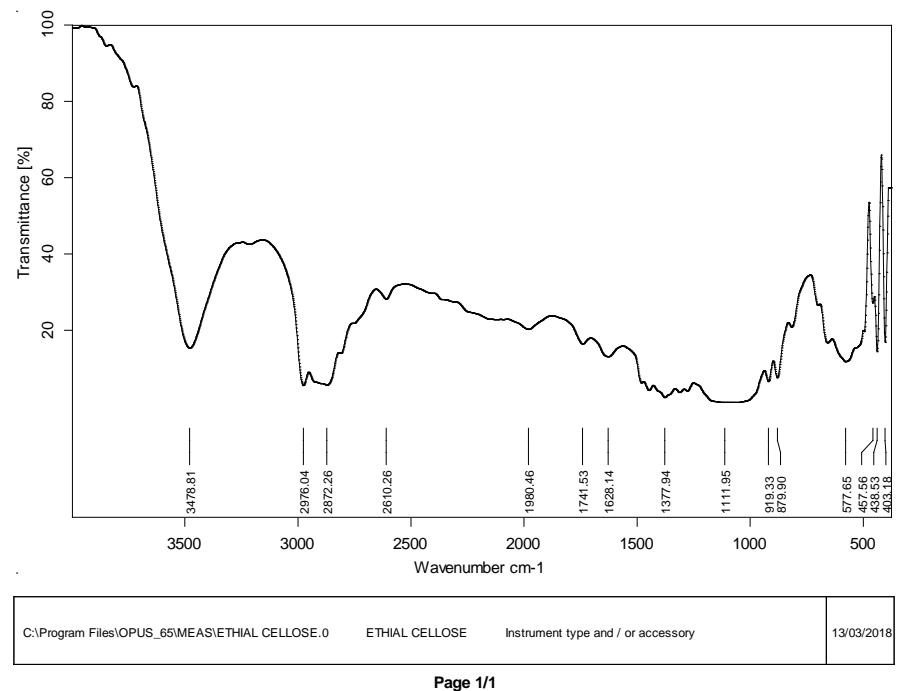

Ethyl cellulose (5b)

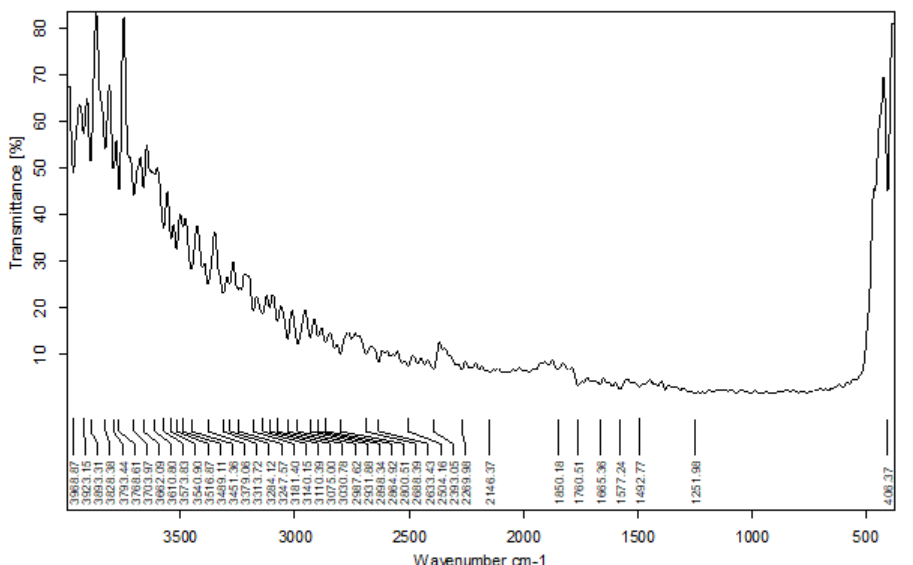

Quercetin loaded nanosponges (5c)

Fig. (5a, 5b, 5c): Fourier transform infrared spectroscopy

\section{In vitro release studies}

In vitro release studies were done by using a phosphate buffer of $\mathrm{pH}$ 7.4. All the formulations of nanosponges were subjected to in vitro drug release studies by using a dialysis membrane. The formulation
F4 has shown the maximum cumulative percentage of drug release 98.6\% for $7 \mathrm{~h}$; the $\mathrm{F} 4$ has shown the highest release in $7 \mathrm{~h}$ when compared to all other formulations. The cumulative drug release profile was obtained by taking time on $\mathrm{x}$-axis and \% cumulative percent of drug release on the y-axis [11]. The release studies were 
performed for all the formulations F0 (Pure drug), F1, F2, F3, F4, F5. The amount of drug release was found to be $98 \%$ for formulation F4 in $7 \mathrm{~h}$, for $\mathrm{F} 3$ it is $90.5 \%$ in $6 \mathrm{~h}, \mathrm{~F} 2 \mathrm{70 \%}, \mathrm{F} 163.5 \%$, F5 79.4\% and pure drug $39 \%$ at the end of $7 \mathrm{~h}$. When compared from F0-F5, the F4 has shown the highest drug release when compared to all other formulations this may be accredited because of the high solubility of the drug. The optimized F4 formulation followed the zero-order drug release profile. Percentage cumulative drug release profile from F0-F5 was given fig. 6 . The concentration of polymer played an important role in the release characteristics.

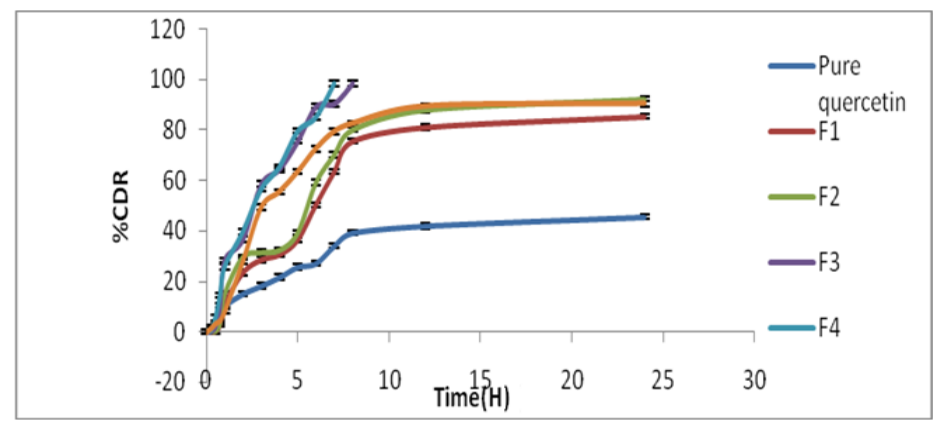

Fig. 6: Comparative in vitro dissolution profile of different quercetin loaded nanosponges formulations, mean $\pm S D, n=3$

\section{Characterization of quercetin-loaded nanosponges of gels}

The prepared gels were homogenous and they exhibit no aggregates, they were transparent in nature and smooth in appearance. The physicochemical properties like $\mathrm{pH}$, viscosity and drug content of the formulated gels were calculated and tabulated in table 4 [9]. All the prepared gels from X1-X6 has shown a $\mathrm{pH}$ range of 6.8-7.0. This is considered as acceptable ranges to reduce the skin irritation. The viscosity of the gels plays an important role in the extrudability and the release of the drug. Gels with high viscosity may not extrude that easily from the tube and low viscous gels may flow easily from the tube that is the reason there should be an optimum viscosity for the gels. The viscosity of the formed gels ranges from 8,300-48,700cps, the viscosity variation was due to change in the concentrations of the polymers (carbapol934 and hydroxypropyl methylcellulose) as the concentration of the polymer hydroxyl propyl methyl cellulose increased there was an increase in the viscosity of the gel. The drug content of the gels from X1X6 was found to be in the range $95-98 \%$ and given in table 4 .

Table 4: Evaluation of physicochemical properties of quercetin loaded nanosponges gel

\begin{tabular}{lllll}
\hline S. No. & Carbopol 934:HPMC & Viscosity (cps) & Appearance & Drug content (\%) \\
\hline 1 & X1 & 8,300 & Homogenous & $98.5 \pm 0.69$ \\
2 & X2 & 8,900 & Homogenous & $96.5 \pm 1.38$ \\
3 & X3 & 10,200 & Homogenous & $95.2 \pm 0.87$ \\
4 & X4 & 24,560 & Homogenous & $97.4 \pm 3.54$ \\
5 & X5 & 48,745 & Homogenous & $94.2 \pm 2.5$ \\
6 & X6 & 8,640 & Homogenous & $96.2 \pm 0.53$ \\
\hline
\end{tabular}

The values are given as mean $\pm \mathrm{SD}, \mathrm{n}=3$

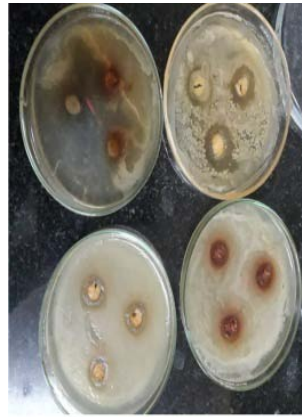

Staphylo coccus aureus

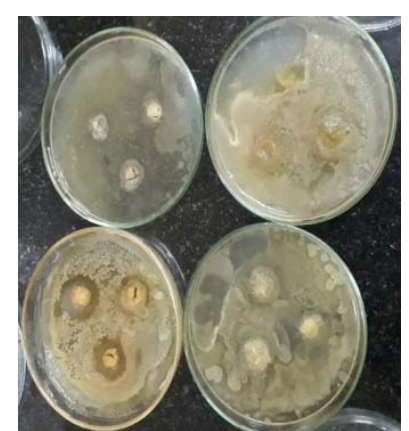

Bacillus subtilis

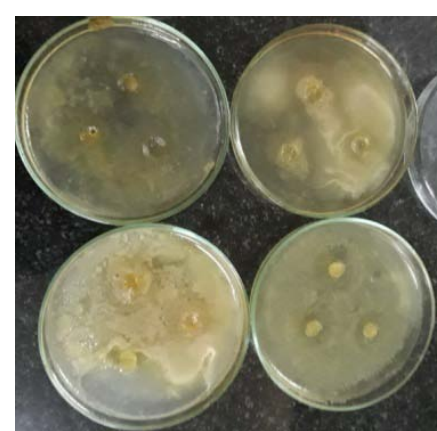

Proteous vulgaris

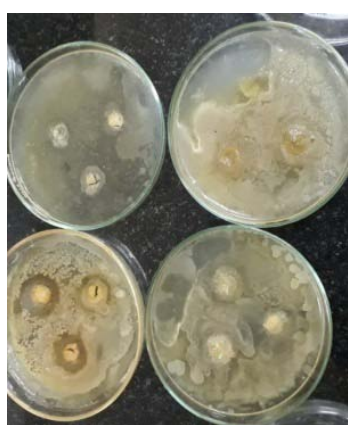

Escherechia coli

Fig. 7: Antimicrobial effect of quercetin-loaded nanosponges gel against bacteria Staphylococcus aureus, Bacillus subtilis, Proteus vulgaris, Escherichia coli studies by a zone of inhibition method

\section{Antimicrobial activity by a zone of inhibition method}

This study was determined by using the bacterial strains Staphylococcus aureus, Escherichia coli, Proteus vulgaris, Bacillus subtilis obtained from HiMedia. The antimicrobial activity was carried out for optimized formulation X1 quercetin loaded nanosponges gel [13]. The gel showed that they were microbiologically inert towards the bacterial strains. The zone of inhibition was reported for Staphylococcus aureus was $2.9 \mathrm{~cm}^{2}$, Escherichia coli was $3.2 \mathrm{~cm}^{2}$, Proteus vulgaris was $3.0 \mathrm{~cm}^{2}$ and Bacillus subtilis was $3.4 \mathrm{~cm}^{2}$ were represented in fig. 7 .

\section{Ex-vivo skin permeation studies and flux value determination}

The quercetin loaded nanosponges gel and pure quercetin gel were subjected to ex-vivo skin permeation studies using goatskin. The amount of drug permeated through the goat skin into the receptor compartment was measured by taking the samples at regular intervals of time [32]. Among all the formulations, the cumulative percent of drug permeated through the skin for the optimized X1 gel loaded with F4 formulation was found to be $95 \%$ for four hours and the cumulative percentage of drug release for pure quercetin gel X6 it showed $50 \%$ for $24 \mathrm{~h}$. The cumulative percent of drug diffused was given in (fig. 8). Highest ex-vivo skin permeation from X1 may be 
attributed due to the increased solubility of the drug within the gel matrix into the test media. The permeation parameters were calculated from a graph by plotting the cumulative percent drug release on the $y$-axis versus time on the $x$-axis. The flux was calculated from the slope of the graph and was found as $18.4 \mu \mathrm{g} / \mathrm{cm}^{2} / \mathrm{h}$ for quercetin nanosponges and $10.3 \mu \mathrm{g} / \mathrm{cm}^{2} / \mathrm{h}$ for pure quercetin and the flux value was more for quercetin nanosponges than pure quercetin gel. The lag time, permeation coefficient, diffusion coefficient was calculated for quercetin nanosponges gel and pure celecoxib gel and the values are as follows $(0.03,0.98 \mathrm{~h})(3.12,2.1 \mathrm{~kg})\left(0.0234,0.16 \mathrm{~mol} / \mathrm{m}^{3}\right)$. The data obtained from the ex-vivo skin permeation studies were subjected to the kinetic data modeling to find out the release mechanism studies from the quercetin loaded nanosponges gel. From the fig. 8 and it was found that the nanosponges loaded quercetin gel release follows zero-order mechanism which governs the release of the drug. From the equation $\mathrm{R}^{2}=0.99$, the drug release follows the Higuchi model which indicates it follows the diffusion mechanism (fickian $\mathrm{n}<0.5$ ). The quercetin loaded nanosponges gel showed the best release when compared to pure quercetin gel.

\section{Stability studies}

The studies were performed as per ICH guidelines for the optimized F4 formulation which was converted into a polymer-based topical gel.
The physical appearance of this gel was observed it does not show any variations and they appear smooth, uniform distribution without any aggregate formation for a period of three months. This implies that the formed quercetin loaded nanosponges gel and pure quercetin gel were stable. The percentage of drug content, in vitro drug release studies, shows no significant change in the drug content and percent of drug release. It was concluded that optimized formulation (X1) and pure quercetin gel were stable during the period of 3 mo of stability studies. The results were tabulated in table 5 .

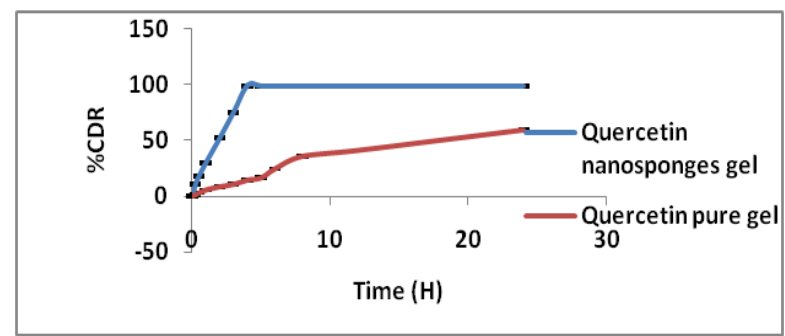

Fig. 8: Ex-vivo skin permeation studies of pure quercetin gel and quercetin loaded nanosponges gel

Table 5: Stability studies for the optimized quercetin loaded nanosponges gel

\begin{tabular}{|c|c|c|c|c|c|c|c|c|}
\hline Temperatures & $25 \pm 2{ }^{\circ} \mathrm{C}$ & & & & $40 \pm 2{ }^{\circ} \mathrm{C}$ & & & \\
\hline Time in days & pH & $\begin{array}{l}\text { Drug } \\
\text { content }\end{array}$ & Homogeneity & $\begin{array}{l}\text { In vitro drug } \\
\text { release }\end{array}$ & pH & $\begin{array}{l}\text { Drug } \\
\text { content }\end{array}$ & Homogeneity & $\begin{array}{l}\text { In vitro drug } \\
\text { release }\end{array}$ \\
\hline $\begin{array}{l}\text { Freshly } \\
\text { prepared }\end{array}$ & $7.0 \pm 0.1$ & 98.5 & $\begin{array}{l}\text { Smooth and } \\
\text { transparent }\end{array}$ & $98.6 \%$ & $7.0 \pm 0.2$ & 97.5 & $\begin{array}{l}\text { Smooth and } \\
\text { transparent }\end{array}$ & $98.6 \%$ \\
\hline $30 \mathrm{~d}$ & $7.0 \pm 0.2$ & 98.5 & $\begin{array}{l}\text { Smooth and } \\
\text { transparent }\end{array}$ & $97.2 \%$ & $7.0 \pm 02$ & 97.5 & $\begin{array}{l}\text { Smooth and } \\
\text { transparent }\end{array}$ & $97.2 \%$ \\
\hline $60 \mathrm{~d}$ & $6.9 \pm 0.1$ & 97.8 & $\begin{array}{l}\text { Smooth and } \\
\text { transparent }\end{array}$ & $97.2 \%$ & $6.8 \pm 02$ & 96.9 & $\begin{array}{l}\text { Smooth and } \\
\text { transparent }\end{array}$ & $97.2 \%$ \\
\hline $90 \mathrm{~d}$ & $6.8 \pm 0.2$ & 97.6 & $\begin{array}{l}\text { Smooth and } \\
\text { transparent }\end{array}$ & $97.0 \%$ & $6.7 \pm 0.3$ & 96.9 & $\begin{array}{l}\text { Smooth and } \\
\text { transparent }\end{array}$ & $97.0 \%$ \\
\hline
\end{tabular}

The values are given as mean $\pm S D, n=3$.

\section{Pharmacodynamic studies}

Anti-inflammatory activity was carried out using carrageenaninduced paw edema method. For quercetin loaded nanosponges gel and pure quercetin gel. For this study male, Wistar albino rats were selected which were grouped into five, each group containing six animals, weighing $150-200 \mathrm{~g}$. The anti-inflammatory test results are as follows the percentage inhibition of paw volume for Group III was $76.2 \%$, Group IV $40.4 \%$ and Group V $96.4 \%$ which is indicated in the fig. 9. From the results, it is evident that there is a significant difference $\left({ }^{*} \mathrm{p}<0.005\right)$ between the pure quercetin gel and quercetin loaded nanosponges gel. The data were subjected to ANOVA (Graph Pad Prism, software), it was found that the F ratio was 3.0 (fc $<\mathrm{ft}$ ). So there is a significant difference between the pure quercetin and quercetin loaded nanosponges gel. Quercetin loaded nanosponges gel shows effective inhibition when compared to the marketed gel and pure quercetin gel (quercetin loaded nanosponges gel>marketed gel>pure quercetin gel).

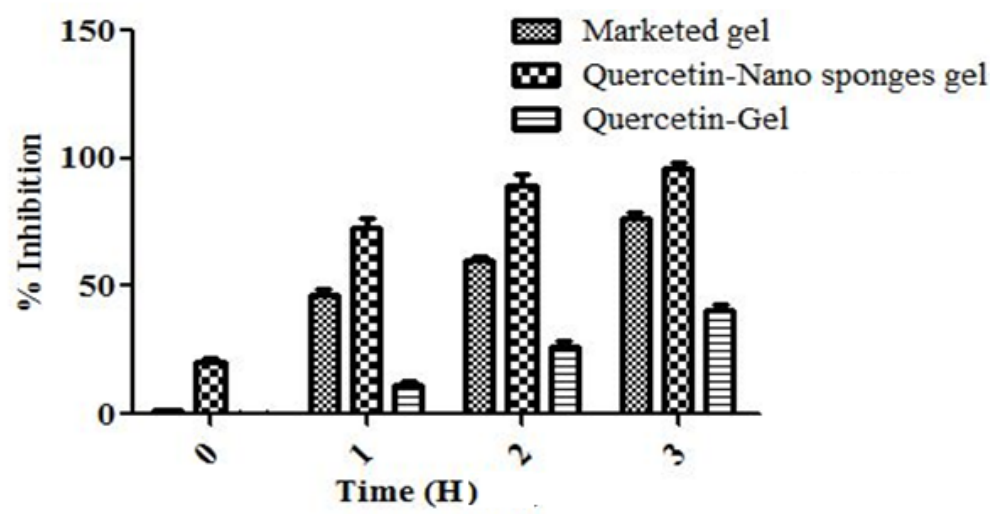

Fig. 9: Percent edema inhibition of quercetin loaded nanosponges gel, marketed gel and pure quercetin gel, $\mathrm{P}<0.05$ compared with disease control values were expressed as mean \pm SEM observation, ANOVA $\left(F C_{>} \mathrm{f}_{\mathrm{t}}\right.$ ) there is a significant difference 


\section{Pharmacokinetic study}

The current analytical method as mentioned above was applied for the study of pharmacokinetic parameters of pure quercetin and quercetin loaded nanosponges gel. The chromatogram of quercetin showing retention time of 7.6 having internal standard (glibenclamide) peak at 3.1 was shown in fig. 10. The mean plasma concentration versus time profile of pure quercetin gel and quercetin loaded nanosponges gel after topical administration of both the gels were summarized as follows $C_{\text {max }}(15.5,27.05 \mu \mathrm{g} / \mathrm{ml})$,
$\mathrm{T}_{\max }(4,2 \mathrm{~h}), \mathrm{t}_{1 / 2}(10,9.7 \mathrm{~h})$, AUC $(90,126 \mu \mathrm{g} / \mathrm{ml})$ as follows. The mean plasma concentration (AUC), $\mathrm{C}_{\max , \mathrm{T}} \mathrm{T}_{\max } \mathrm{t}_{1 / 2}$ was statistically significant $\left({ }^{*} \mathrm{p}<0.05\right)$ when compared to the pure quercetin gel and quercetin loaded nanosponges gel. Pure quercetin gel and quercetin loaded nanosponges gel which is given fig. 11. Quercetin loaded nanosponges gel has shown fast absorption with $\mathrm{C}_{\max }(27.05 \mu \mathrm{g} / \mathrm{ml})$ and more AUC $(126 \mu \mathrm{g} / \mathrm{ml})$ as it contains the tiny size of nanosponges $(188.3 \mathrm{~nm})$ which makes the drug more permeable when compared to pure quercetin gel through the skin.

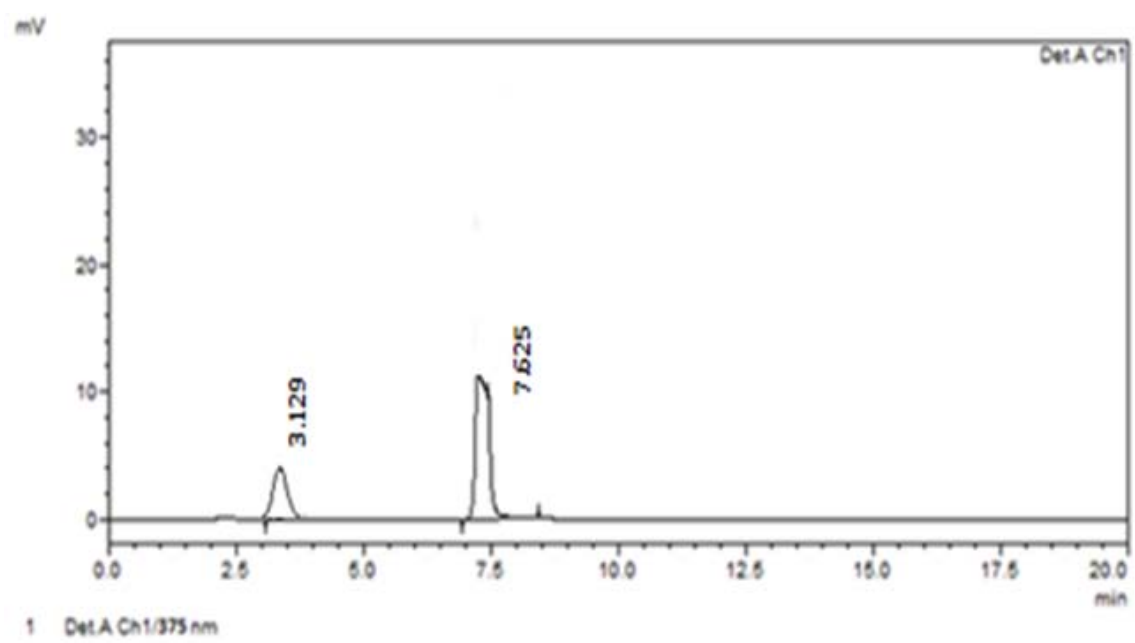

Fig. 10: HPLC chromatogram of quercetin

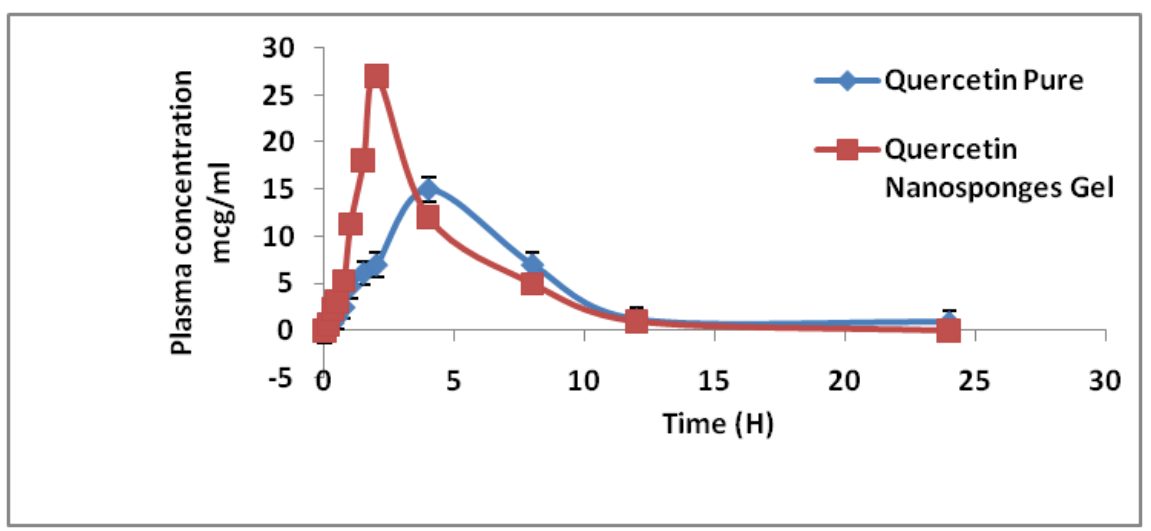

Fig. 11: Plasma concentration time profile of pure quercetin gel and quercetin nanosponges gel in rats $n=6$, Data presented as mean \pm SD

\section{CONCLUSION}

From above all the results it can be concluded that the nanosponges prepared with ethyl cellulose and drug quercetin by emulsion solvent diffusion method were effectively incorporated into the topical gel. The quercetin loaded nanosponges gel showed more for anti-inflammatory activity when compared to pure quercetin gel and marketed Voveran Emulgel.

\section{AUTHORS CONTRIBUTIONS}

All the authors have contributed equally

\section{CONFLICT OF INTERESTS}

Declared none

\section{REFERENCES}

1. Soo Nam Park, Hye Jin Lee, Hae Soo Kim, Min A Park, Hyun A $\mathrm{Gu}$. Enhanced transdermal deposition and characterization of quercetin-loaded ethosomes. J Chem Eng 2013;3:688-92.
2. Wang Q, Bao Y, Ahire J, Chao Y. Co-encapsulation of biodegradable nanoparticles with silicon quantum dots and quercetin for monitored delivery. Adv Healthc Mater 2018;3:459-66.

3. Qi Tan, Weidong Liu, Chenyu Guo, Guangxi Zhai. Preparation and evaluation of quercetin-loaded lecithin-chitosan nanoparticles for topical delivery. Int J Nanomed 2011;6:1621-34.

4. Soheirn Abd, El Rehamanan, Suhailal, S-Al Jammeel. Quercetin nanoparticles preparation and characterization. Indian J Drug 2014;2:96-103.

5. Chen Yu G, Chun Fen Y, Qi Lu L. Development of a quercetinloaded nanostructured lipid carrier formulation for topical delivery. Int J Pharm 2012;2:292-8.

6. Gulian Quan, In Pan, Zhihua Wang, Qiao Liu Wu. Linghu did an erratum to lactosaminated mesoporous silica nanoparticles for asialoglycoprotein receptor-targeted anticancer drug delivery. J Nanobiotechnol 2015;3:83-9.

7. Avnesh Kumari A, Sudesh Kumar Yadava, Yogesh B, Pakade B, Bikram Singh, Subhash Chandra Yadava. Development of 
biodegradable nanoparticles for delivery of quercetin. Colloids Surf B Bio Int 2010;80:184-92.

8. Kumari A, Yadav SK, Pakade YB, Singh B, Yadav SC. Development of biodegradable nanoparticles for delivery of quercetin. Colloids Surf B Biointerfaces 2010;80:184-92.

9. SB Han, SS Kwon, YM Jeong, ER Yu, SN Park. Physical characterization and in vitro skin permeation of solid lipid nanoparticles for transdermal delivery of quercetin. Int J Cosmetic Sci 2014;36:588-97.

10. Gurupreet Khandav, DC Bhatt, Deepak Kumar Jindal. Formulation and evaluation of aloplurinol loaded chitosan particles. Int J Appl Pharma 2019;3:49-52.

11. Ansari KA, Vavia PR, Trotta F, Cavalli R. Cyclodextrin based nanosponges for delivery of resveratrol: in vitro characterization, stability, cytotoxicity, and permeation study. AAPS PharmSciTech 2011;129:279-86.

12. Sema Saglin, Ugursalgin, Ozgun Vantansever. Synthesis and characterization of beta-cyclodextrin nanosponge and its application for removal of p-nitrophenol from water. Clean Soil Air Water 2015;45:45-56.

13. Patel J, Trivedi J, Chudhary DS. Microsponges for topical drug delivery. Int J Pharm Res Bio Sci 2014;14:625-38.

14. Swarupa Arvapally, M Harini, G Harshita. Formulation and in vitro evaluation of glipizide nanosponges. Am J Pharm Tech Res 2017;7:12-32.

15. Bettini R, Catellani PL, Santi P, Massimo G, Peppas NA, Colombo $P$. Translocation of drug particles in HPMC matrix gel layer: effect of drug solubility and influence on release rate. J Controlled Release 2001;70:383-91.

16. Cao M, Ren L, Chen G. Formulation optimization and ex vivo and in vivo evaluation of celecoxib micro emulsion-based gel for transdermal delivery. AAPS PharmSciTech 2017;18:60-71.

17. Blanco Garcla E, Otero Espinar FJ, Blanco M Endez J, Leiro Vidal JM, Luzardo Alvarez A. Development and characterization of anti-inflammatory activity of curcumin-loaded biodegradable microspheres with potential use in intestinal inflammatory disorders. Int J Pharm 2017;12:86-100.

18. Yinghui Liu, Changshan Sun, Yanru Hao, Gongying Jiang, Li Zheng, Siling Wang. Mechanism of dissolution enhancement and bioavailability of poorly water-soluble celecoxib by preparing stable amorphous nanoparticles. J Pharm Pharm Sci 2010;13:589-606.

19. Geetha Agarwal, Manjil Nagpal, Gurpreet Kaur. Development and comparison of nanosponge and noisome based gel for topical delivery of tazarotene. Pharma Tech 2016;4:213-28.

20. Jain SK, Chourasia MK, Masuriha R, Soni V, Jain A, Jain NK, et al. Solid lipid nanoparticles bearing flurbiprofen for transdermal delivery. Drug Delivery 2005;12:207-15.

21. Girish Konadalkar, Asish Dev. Design, development, and evaluation of ion-activated in-situ gel of moxifloxacin hydrochloride. Asian J Pharma Clin Res 2019;3:94-103.

22. Liu Y, Sun C, Hao Y, Jiang T, Zheng L, Wang S. Mechanism of dissolution enhancement and bioavailability of poorly watersoluble celecoxib by preparing stable amorphous nanoparticles. J Pharm Pharm Sci 2010;13:589-606.

23. Biswarajan Mohanthy Dipak K, Mujumdar Sagar, MIshra Amulya K Panda. Development and characterization of itraconazole loaded solid lipid nanoparticles. Pharm Dev Tech 2015;20:458-64.

24. Patil Bhagyashree Subhash, SK Mohite. Formulation design and development of artesunate nanosponges. Eur J Pharma Med Res 2016;3:206-11.

25. S Jaya, S Divas. Formulation and in vitro evaluation of matrix tablets of metoclopramide hydrochloride. Int J Appl Pharm 2018;11:25-30.

26. M Tuncay, S Calis, HS Kas M. In vitro and in vivo evaluation of diclofenac sodium loaded albumin microspheres. J Microencapsulation 2000;17:145-55.

27. Ehab A Found, Alaa Eldeen B Yassin, Hamdan N Alabama. Characterisation of celecoxib loaded solid lipid nanoparticles formulated with tristearin and soft is a 100. Trop J Pharm Res 2015;14:205-10.

28. Karade P. Formulation, and evaluation of celecoxib gel. J Drug Delivery Ther 2012;2:132-5.

29. Azadeh Serri, Arash Mahbouli, Afshin Zarghi, Hamid R Moghimi. PAMAM-dendrimer enhanced the antibacterial effect of vancomycin hydrochloride against gram-negative bacteria. J Pharm Pharm Sci 2019;22:10-21.

30. Abhishek D Dorle, Kumar S Swami, Sujith K Nagare, Supriya R Hyam. Design and evaluation of novel topical gel of tinospora cordifolia as antimicrobial agent Asian J Pharm Clin Res 2015;69:24-34.

31. Khalid A Anasari, Pradeep R Valli, Franscisco Trota. Roberta cavalla cyclodextrin based nanosponges for delivery of resebertol: in vitro characterization, stability, cytotoxicityand permeation study. AAPS PharmSciTech 2011;12:279-86.

32. G Jilisha, Vidya Viswanath. Nanosponge loaded hydrogel of cephalexin for topical delivery. Int J Pharm Sci Res 2015;6:2742.

33. Lalith Kumar. Novel ethosomal gel of clove oil for treatment of cutaneous candidiasis. J Cosme Dermat 2018;5:21-38.

34. Koçkaya EA, Selmanoglu G, Kısmet K, Akay MT. Pathological and biochemical effects of therapeutic and supratherapeutic doses of celecoxib in wistar albino male rats. Drug Chem Toxicol 2010;33:410-4

35. Auda SH, El-Rasoul SA, Ahmed MM, Osman SK, El-Badry M. In vitro release and in vivo performance of tolmetin from different topical gel formulations. J Pharma Invest 2015;45:311-7.

36. S Halirfroosh KO. Assessment of celecoxib poly (lactic-glycolic) acid nanoformulation on drug pharmacodynamics and pharmacokinetics in rats. Eur Rev Med Pharmacol Sci 2016;20:4818-29.

37. L Nikita Sanghavi, SD Bhosale, Yashwant Malode. RP-HPLC method development and validation of quercetin isolated from the plant tridax procumbens. J Sci Innov Res 2014;3:594-7.

38. Guichen Baohua Hao, Dahong JU. Pharmacokinetic and pharmacodynamic study of triptolide loaded liposome hydrogel patch under microneedles on rats with collageninduced arthritis. APSB 2015;5:569-76.

39. Hammam A Mowafy, Fars K Alanazi, Gamal M El Magahraby. Development and validation of an HPLC-UV method for the quantification of carbamazepine in rabbit plasma. Sau Pharm J 2012;3:29-34. 